\title{
The contribution of HIV point-of-care tests in early HIV diagnosis: community-based HIV testing monitoring in Catalonia, 1995 to 2018
}

Laura Fernàndez-López ${ }^{1,2}$, Juliana Reyes-Urueña ${ }^{1,2}$, Anna Conway ${ }^{1,3}$, Jorge Saz ${ }^{4}$, Adriana Morales ${ }^{5}$, Jaime Quezadas ${ }^{6}$, Jordi

Baroja $^{7}$, Anna Rafel ${ }^{8}$, Ander Pazos9 ${ }^{9}$, Anna Avellaneda ${ }^{10}$, Mercè Meroño ${ }^{11}$, Lorena Andreo ${ }^{12}$, Lluís Romero ${ }^{13}$, Anna Lara ${ }^{14}$, Araceli Otón $^{15}$, Benet Rifà ${ }^{16,17}$, Rosa Mansilla ${ }^{16,17}$, Joan Colom ${ }^{17}$, Jordi Casabona ${ }^{1,2,18}$

1. Centre for Epidemiological Studies on Sexually Transmitted Infections and HIV/AIDS of Catalonia (CEEISCAT), Catalan Health Department, Barcelona, Spain

2. Centros de Investigación Biomédica en Red Epidemiología y Salud Pública (CIBERESP), Spain

3. The Institute for Health Science Research Germans Trias i Pujol (IGTP), Badalona, Spain

4. BCN Checkpoint, Barcelona, Spain

5. Stop Sida, Barcelona, Spain

6. Associació Ciutadana Antisida de Catalunya (ACASC), Barcelona, Spain

7. Centre Jove d’Atenció a les Sexualitats (CJAS), Barcelona, Spain

8. Associació Antisida de Lleida, Lleida, Spain

9. Gais Positius, Barcelona, Spain

10. Actuavallès, Sabadell, Spain

11. Fundació Àmbit Prevenció, Barcelona, Spain

12. CAS/ARD Lluís Companys, Creu Roja Barcelona, Barcelona, Spain

13. Assexora'Tgn (Associacio Comunitària de Salut Sexual del Camp de Tarragona), Tarragona, Spain

14. Associació Comunitària Anti Sida de Girona (ACAS), Girona, Spain

15. Creu Roja Tarragona, Tarragona, Spain

16. Section for Surveillance, Prevention and Control of Sexually Transmitted Infections and HIV, Public Health Agency of Catalonia, Barcelona, Spain

17. Programme for Prevention, Control and Treatment of HIV, STIs and Viral Hepatitis, Public Health Agency of Catalonia, Barcelona, Spain

18. Department of Paediatrics, Obstetrics and Gynaecology, and Preventive Medicine, Universitat Autònoma de Barcelona, Bellaterra, Spain

Correspondence: Laura Fernàndez-López (Iflopez@iconcologia.net)

Citation style for this article:

Fernàndez-López Laura ORCID icon, Reyes-Urueña Juliana ORCID icon, Conway Anna, Saz Jorge, Morales Adriana, Quezadas Jaime, Baroja Jordi , Rafel Anna , Pazos Ander, Avellaneda Anna, Meroño Mercè, Andreo Lorena, Romero Lluís, Lara Anna, Otón Araceli , Rifà Benet, Mansilla Rosa, Colom Joan , Casabona Jordi. The contribution of HIV point-of-care tests in early HIV diagnosis: community-based HIV testing monitoring in Catalonia, 1995 to 2018 . Euro Surveill. 2020;25(43): pii=1900424. https://doi.org/10.2807/1560-7917.ES.2020.25.43.1900424

Background: Community-based HIV testing services combined with the use of point-of-care tests (POCT) have the potential to improve early diagnosis through increasing availability, accessibility and uptake of HIV testing. Aim: To describe community-based HIV testing activity in Catalonia, Spain, from 1995 to 2018, and to evaluate the impact of HIV POCT on the HIV continuum of care. Methods: A community-based network of voluntary counselling and testing services in Catalonia, Spain has been collecting systematic data on activity, process and results since 1995. A descriptive analysis was performed on pooled data, describing the data in terms of people tested and reactive screening test results. Results: Between 1995 and 2018, 125,876 HIV tests were performed (2.1\% reactive). Since the introduction of HIV POCT in 2007, a large increase in the number of tests performed was observed, reaching 14,537 tests alone in 2018 (1.3\% reactive). Men who have sex with men (MSM), as a proportion of all people tested, has increased greatly over time reaching $74.7 \%$ in 2018 . The highest percentage of reactive tests was found in people who inject drugs followed by MSM. The contribution of community-based HIV testing to the overall total notified cases in the Catalonia HIV registry has gradually increased, reaching $37.9 \%$ in 2018 , and $70 \%$ of all MSM cases. In 2018 , the percentage of individuals with a reactive screening test who were linked to care was $89.0 \%$. Conclusion: Our study reinforces the important role that communitybased HIV POCT has on the diagnosis of HIV in key populations.

\section{Introduction}

In recent years, efforts to reach the 90-90-90 targets (90\% of all people living with HIV knowing their HIV status, $90 \%$ of all people diagnosed with HIV receiving antiretroviral therapy and $90 \%$ of all people receiving antiretroviral therapy having viral suppression) advocated by the Joint United Nations Programme on HIV/ AIDS (UNAIDS), have led to an improvement in accessibility and coverage of testing programmes. This, in turn, has reduced the number of people living with 
undiagnosed HIV infection and increased early diagnoses [1]. Monitoring and evaluation (M\&E) is an essential component of any effective testing programme. While strategic information should guide the design of testing initiatives, M\&E permits continuous evaluation of targets and programme effectiveness, efficiency and impact. Such data can prove invaluable in planning improvements [2].

Catalonia is an autonomous community located in the north-east of Spain. In 2018, it had a population of $7,543,825$ inhabitants. This region has a low-level HIV epidemic, where high levels of infection are found only in specific groups, particularly men who have sex with men (MSM). As of 31 December 2017, Catalonia had a rate of 8.1 HIV diagnoses per 100,000 inhabitants, with $53.6 \%$ of all diagnoses in 2017 attributed to MSM [3].

In Catalonia, HIV testing M\&E forms part of the Integrated AIDS/HIV/STI Surveillance System of Catalonia (SIVES) [4] and is based on two main sources of information: (i) the network of public hospital laboratories, primary healthcare centre laboratories and private laboratories (HIVLABCAT), which have voluntarily reported diagnostic HIV testing and results since 1992; and (ii) the network of community-based voluntary counselling and testing (CBVCT) services, which has offered free, anonymous, voluntary and confidential counselling and testing since 1995 [5]. This report will focus on the data collected by the CBVCT network.

CBVCT services are considered an effective strategy for HIV testing, especially for key populations $[6,7]$, and have expanded in the European Union/European Economic Area (EU/EEA) since 2010 through a variety of service delivery models [8]. This strategy has been proven to increase the availability, accessibility and uptake of HIV testing in order to reduce the number of people who do not know their HIV status or who are diagnosed late [9], impacting the first 90 target set by UNAIDS [10]. In addition, this strategy increases the proportion of first-time testers, increases the proportion of participants who undertook follow-up $C_{4}$ tests after diagnosis, detects patients at an earlier stage of infection, increases the number of new HIV diagnoses, and potentially reduces the stigma and discrimination faced by key populations [6].

A systematic review found that the use of HIV pointof-care tests (POCT) as part of CBVCT interventions, combined with behavioural interventions either at individual or community level, has the potential for enormous impact on the HIV epidemic [11]. Scaling up the CBVCT service model was thought to increase the likelihood of achieving the 90-90-90 target by 2020 [12], but the scale up in Europe has been impacted by limited funding, poor integration with national HIV programmes and regulatory barriers. There is a need for guidance to address these implementation challenges, including $\mathrm{M \& E}$, and a need to assist countries in developing, implementing and evaluating national policies [13].

Community-based testing started in Catalonia with only a few sites offering traditional testing, where a nurse was required to perform venepuncture and send the blood sample to a laboratory. Traditional testing was replaced with HIV POCT in 2007, which allowed the expansion of testing programmes in the community. Since 2007, more sites have been offering HIV POCT, and the number of tests performed has increased exponentially [5].

Catalonia has experience in the scaling up of CBVCT interventions using HIV POCT with linkage to care, support and treatment services, within a solid M\&E framework. Here, our aims are to describe HIV testing activity among those CBVCT services participating in the DEVO (an abbreviation of 'voluntary detection' in Catalan) network from 1995 to 2018 in order to evaluate HIV POCT contribution in the HIV continuum of care.

\section{Methods}

\section{Settings}

In 1995, the Catalan Health Department (currently, the Public Health Agency of Catalonia, ASPCAT) funded a network of CBVCT services to offer free, voluntary and confidential HIV testing in the region. The purpose of the DEVO network was to complement existing facility-based HIV testing. The DEVO network has since expanded from four CBVCT services in 1995 to the current 12 (becoming six organizations in 2001, seven in 2003, eight in 2004, 10 in 2008 and 12 in 2010), mainly operated by NGOs and serving the general population or, in some cases, key populations: MSM, sex workers (SW), young people (under 30 years old), and people who inject drugs (PWID). The participating organisations of the network are all CBVCT services providing HIV testing by trained lay providers through community and outreach services. In addition to providing HIV testing, most organisations perform syphilis and hepatitis $C$ testing and additional HIV prevention activities. One of the organisations also offers other STI tests. Peers and other lay providers have been trained to perform and interpret rapid diagnostic tests with finger-prick blood samples.

Every person who receives a preliminary reactive test is referred to a laboratory or to an infectious disease specialist who conducts a confirmatory test. The diagnosed clients are then linked with appropriate specialist services.

\section{Data collection}

The DEVO network has been collecting systematically standardized data on activity, process and results since 1995. Since 2014, the DEVO network has formed part of the community-based testing (COBATEST) network, a European network of CBVCT services based on the DEVO network experience $[8,14]$. Since then, all 
Number of HIV tests performed ${ }^{\mathrm{a}}$ and percentage of reactive tests by year, Catalonia, Spain, 1995-2018 $(\mathrm{n}=129,117)$

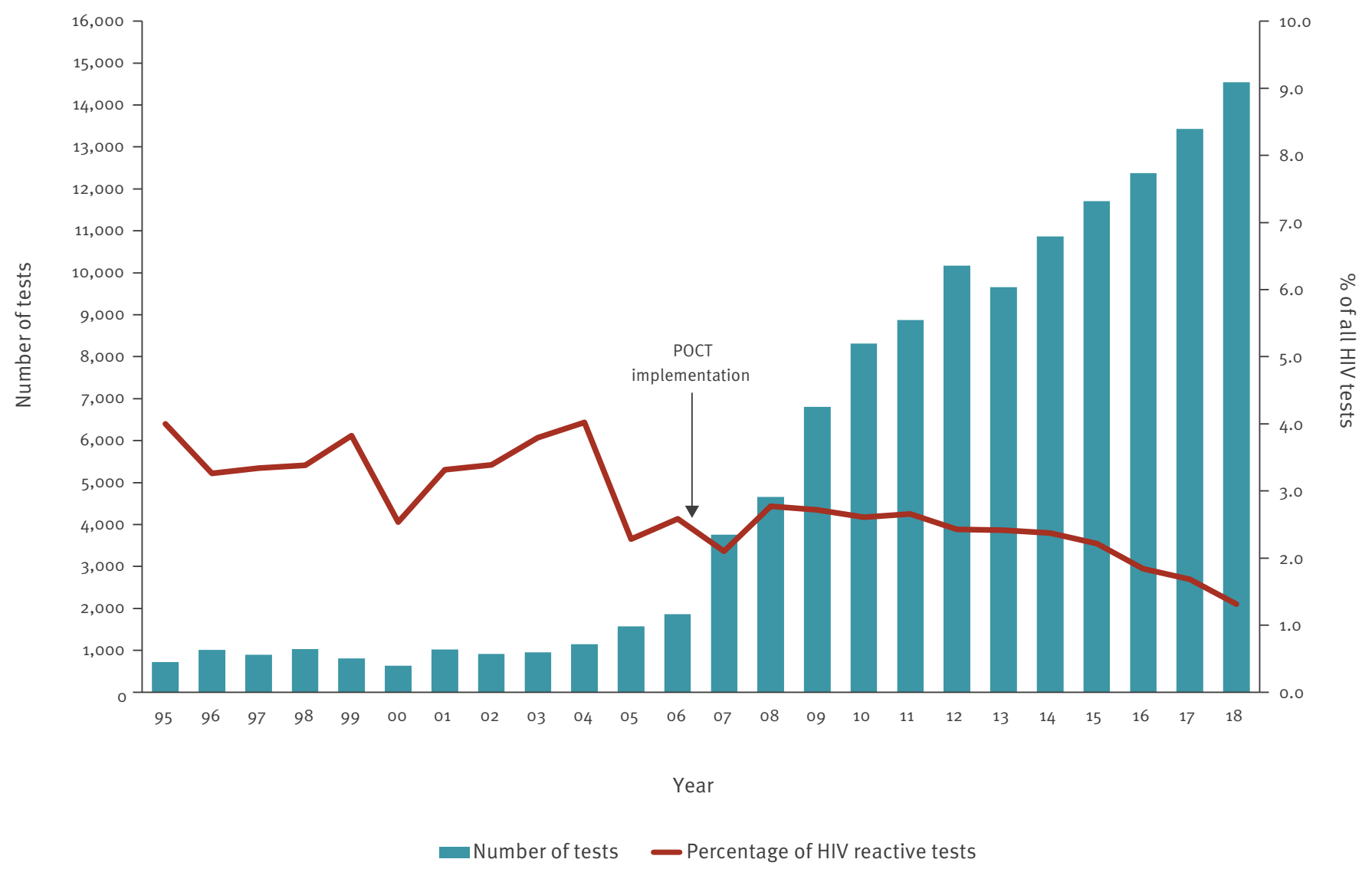

DEVO: voluntary detection; РОCT: point-of-care test.

a Only HIV tests performed within the DEVO network were included.

except one of the CBVCT services use the COBATEST data collection tool (which is based on the DEVO data collection tool) and COBATEST web-based data entry tool through which data can be extracted and analysed in collaboration with the Centre for Epidemiological Studies on Sexually Transmitted Infections and HIV/ AIDS of Catalonia (CEEISCAT) as part of the Public Health Agency of Catalonia (ASPCAT). One of the CBVCT services uses their own data collection tool, and shares the minimum agreed data with CEEISCAT. For monitoring and evaluation purposes, the network currently uses the standardised core indicators defined in the COBATEST network [15], aligned with UNAIDS, World Health Organization (WHO) and European Centre for Disease Prevention and Control (ECDC) recommendations [16-18].

Data collected in the DEVO network include basic demographic information on the tester, test location, testing history, risk behaviour and results of HIV, syphilis and hepatitis C testing. Since 2014 , services in the DEVO network have used a unique identifier for each client, ensuring anonymity while allowing the identification of repeat testers and recording the correct number of individuals tested.

\section{Test used}

From 1995 to 2007, a conventional laboratory test with phlebotomy was used, from 2007 to 2012 the Determine HIV $-1 / 2$ rapid test (Abbott Laboratories, Abbott Park, IL, United States) was used, and since 2012 the new Alere Determine HIV-1/2 Ag/Ab Combo (Abbott Laboratories) test has been used. With both POCTs, the results were obtained in $15-20 \mathrm{~min}$ (15 min for Determine HIV-1/2 rapid test and 20 min for Alere Determine HIV-1/2 Ag/Ab Combo), and test accuracy is very high (Determine HIV-1/2 rapid test: sensitivity 99.6\% (95\% Cl: 99.2-99.8), specificity 99.9\% (95\% Cl: 99.8-100.0) [19,20]; Alere Determine HIV-1/2 Ag/Ab Combo: sensitivity $99.9 \%$ (95\% Cl: $99.4-100.0$ ), specificity $99.8 \%$ (95\% Cl: 99.5-99.9) [21].

\section{Data analysis}

The descriptive analysis was performed on pooled data from 1995 to 2018 and included: (i) the whole time period 1995-2018; (ii) each year individually; (iii) the percentage of people tested distributed by gender, 


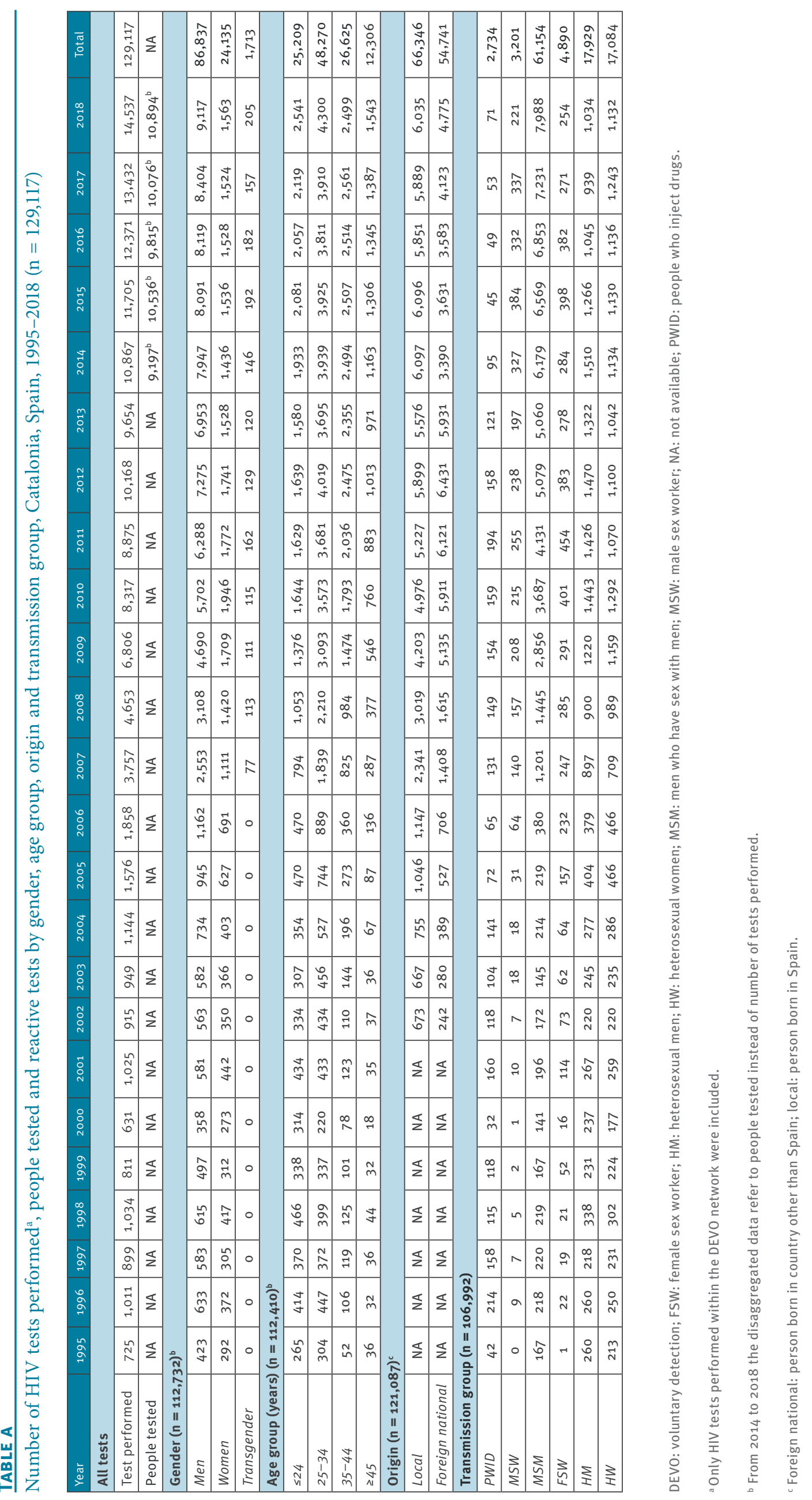



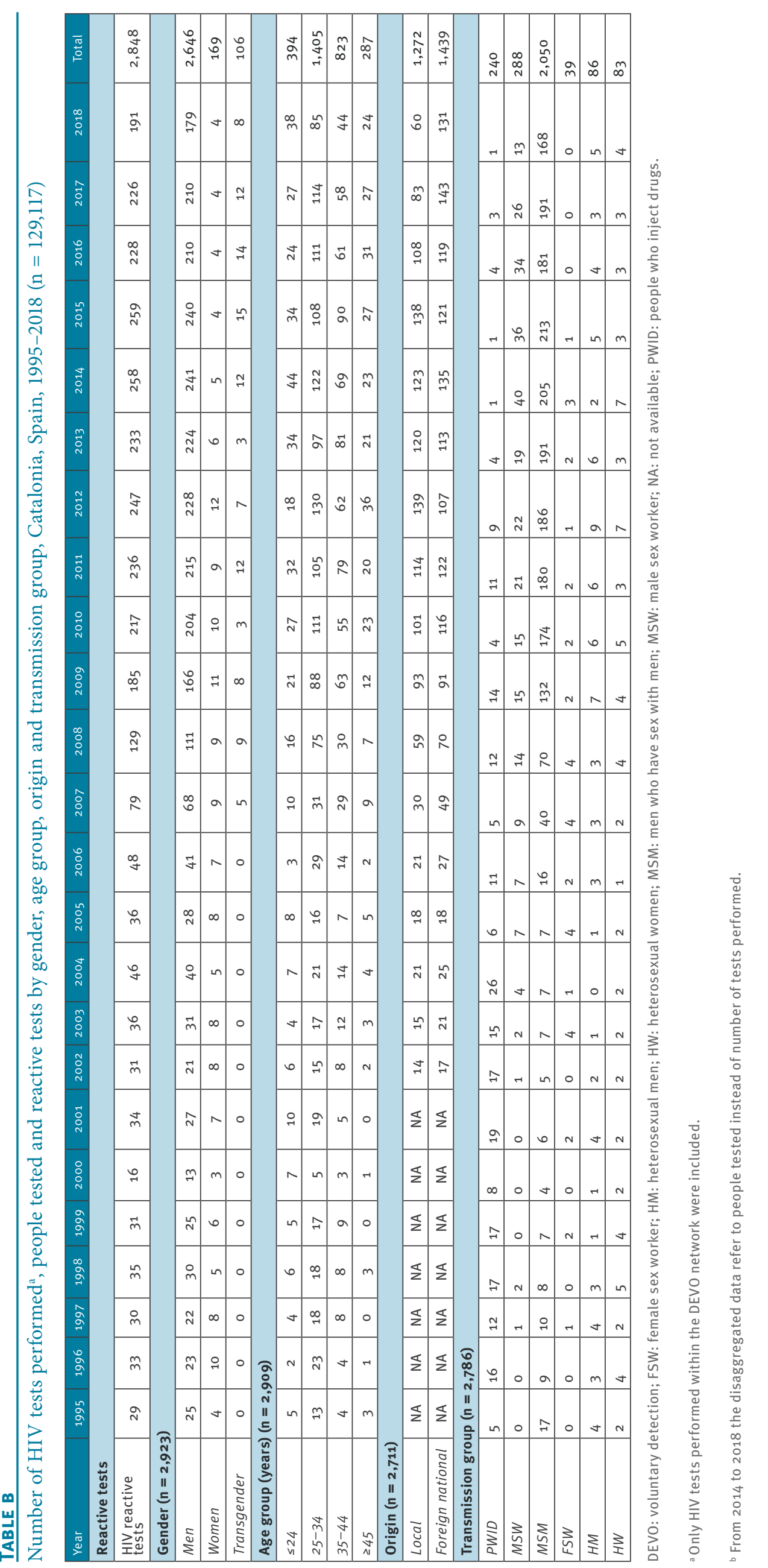
Gender, age, origin and transmission group of (A) all people tested for HIV ( $\left.\mathrm{n}=112,732^{\mathrm{a}}\right)$ and (B) HIV reactive tests (n = 2,848), Catalonia, Spain, 1995-2018

\section{A. People tested}

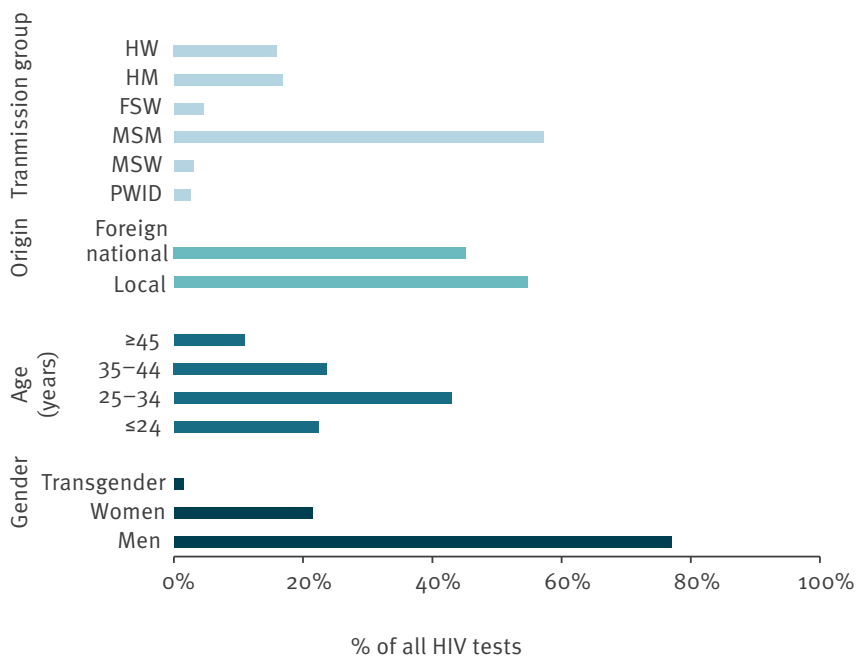

B. HIV reactive cases

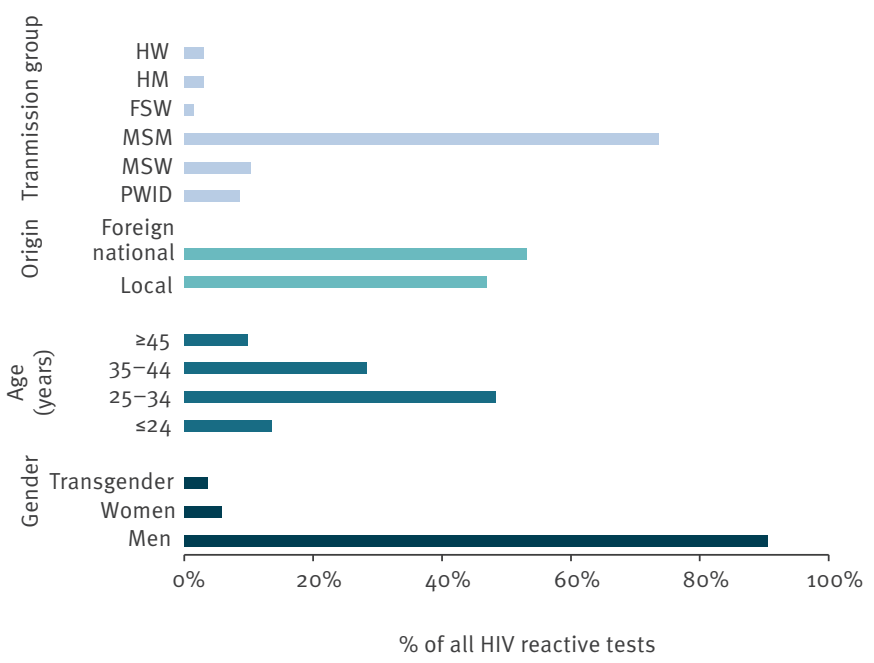

DEVO: voluntary detection; Foreign national: person born in country other than Spain; FSW: female sex worker; HM: heterosexual men; HW: heterosexual women; local: person born in Spain; MSM: men who have sex with men; MSW: male sex worker; PWID: people who inject drugs.

${ }^{\text {a }}$ Only people tested within the DEVO network were included. Before 2014 disaggregated data refers to number of tests instead of people tested.

age, nationality and transmission group; (iv) the percentage of individuals with a confirmed positive test before 2007 and the percentage of individuals with a reactive screening test after 2017 distributed by gender, age, nationality and transmission group. Variables included: gender (men, women, transgender), age, nationality (foreign national defined as born in a country other than Spain, or local defined as born in Spain) and transmission group (constructed as hierarchical, mutually exclusive risk categories in the following order of priority: PWID, male sex workers (MSW), MSM, female sex workers (FSW), heterosexual women (HW), heterosexual men (HM)).

For Figures 4 and 5 the MSW group was added to the MSM group in order to present all the MSM population together.

The lines in Figure 4 chart the evolution of the percentage of reactive screening tests per year by transmission group and were smoothed using the centred moving average method. Using this method, data points were modified four times, each time the average of raw observations at a given point in time was calculated using that point, the one immediately prior and the one immediately after. This method allows for smoothing out short-term fluctuations and highlights long-term trends or cycles [22]. To test trends in Figures 1 and 4, a Pearson's chi-squared test was used.
Linkage to care was defined as 'entry into healthcare or follow-up by a HIV specialist or a HIV unit after a reactive or confirmatory HIV test at a community testing facility' according the definition established in the Euro HIV EDAT project, co-funded by the European Commission [23], and all linkage to care information was collected from patient feedback.

In order to evaluate the contribution of community testing to the total number of diagnosed cases in Catalonia, data from the DEVO network and the Catalonia HIV registry were triangulated, considering that reactive tests detected in the DEVO network were linked to care and therefore were noted in the Catalonia HIV registry. The percentage of HIV cases diagnosed in the community and registered in the Catalonia HIV registry was calculated from 2001 to 2017, the period where Catalonia HIV registry data were available.

All percentages were calculated excluding missing values (which represented less than $5 \%$ ). A p value below 0.001 was considered for statistical significance. Data analysis was performed using PASW Statistics for Windows, version 18.0 (SPSS Inc., Chicago, United States).

\section{Ethical statement}

Ethical approval was not needed at the beginning of the project in 1995, as no biological samples were preserved for the study and data collected from the clients were anonymous and part of the routine services 
Distribution by transmission group of (A) all people HIV tested ${ }^{a}(n=106,992)$ and (B) HIV reactive tests $(n=2,786)$, Catalonia, Spain, 1995-2018

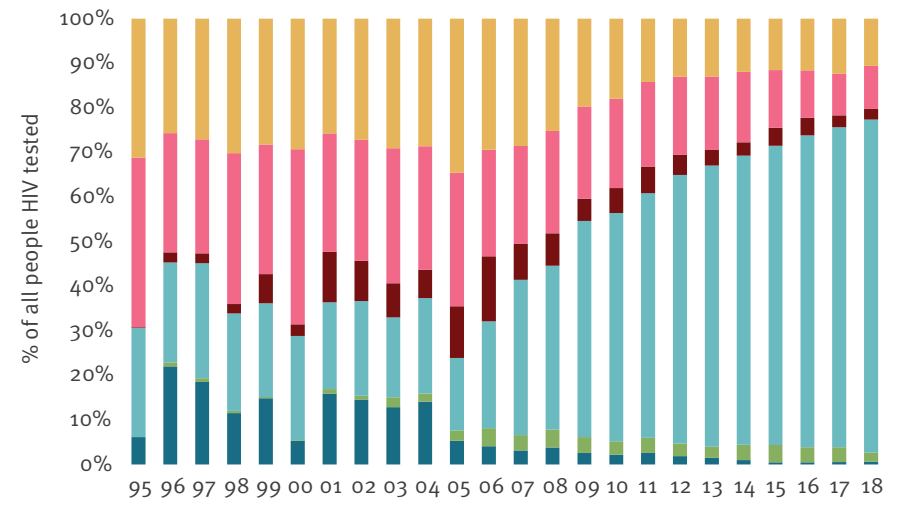

Year

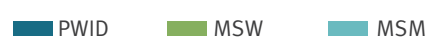

B.

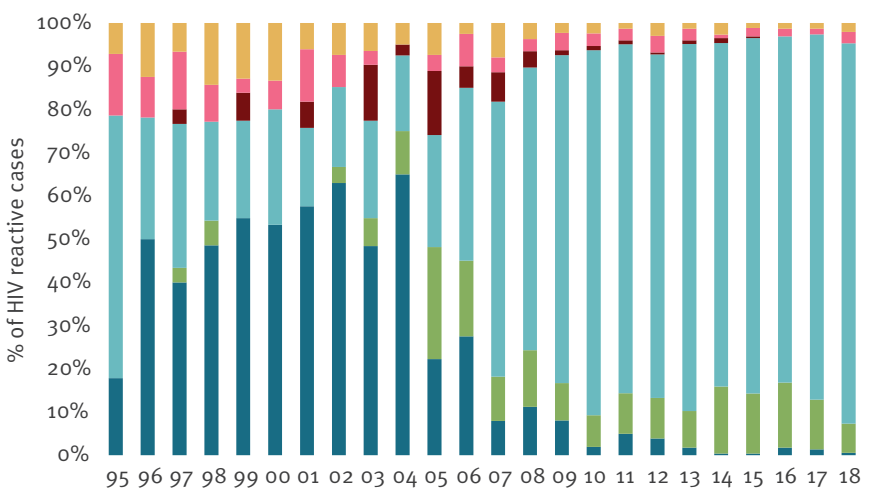

Year

HW

DEVO: voluntary detection; FSW: female sex worker; HM: heterosexual men; HW: heterosexual women; MSM: men who have sex with men; MSW: male sex worker; PWID: people who inject drugs.

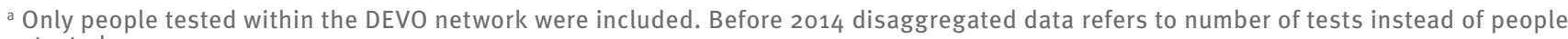
tested.

of CBVCT centres. Since the introduction of POCT, each client taking a POCT gave informed consent stating that they understood the POCT was not a diagnostic test. Since 2014 when the DEVO network joined the COBATEST network, the CBVCT services signed an agreement ensuring they fulfilled the General Data Protection Regulation, where each client has to sign an informed consent explaining the use of the data collected.

\section{Results}

Between 1995 and 2018, 129,117 HIV tests were performed by the DEVO network, of which $2.1 \%$ were reactive. The increase in the number of tests performed by the CBVCT services by year was relatively low until 2006, ranging from 716 in 1995 to 1,849 in 2006 (Figure 1).

With the introduction of the HIV POCTs at the end of 2006 , there was a $102.9 \%$ increase in the number of HIV tests performed in 2007 compared to 2006 (analysis published in a previous study [5]). In 2018, the number of HIV tests peaked at 14,537 , of which $1.3 \%$ $(n=191)$ were reactive. From 2006 to 2018 there was an increase of $686.2 \%$ in number of tests performed (from 1,858 tests performed in 2006 to 14,537 in 2018), with an average annual increase of $21.1 \%$. In the past 10 years, the percentage of reactive tests has been decreasing (statistically significant trend, $p<0.0001$ ), from $2.8 \%(129 / 4,653)$ in 2008 to $1.3 \%(191 / 14,537)$ in 2018.
HIV testing activity differed greatly between CBVCT services, with one organisation, which works only with MSM, performing on average more than half of the total number of HIV tests in the DEVO network.

The Table shows the evolution by year on tests performed, people tested and number of reactive screening tests disaggregated by gender, age group, origin and transmission group.

Between 1995 and $2018,77.0 \%(86,837 / 112,732)$ of the total people tested at the community sites were men, and $92.9 \%(2,646 / 2,848)$ of reactive tests were in men. In men and women, the age group with the most people tested and most reactive tests was 25-34 years old (Figure 2). Foreign nationals accounted for $45.2 \%$ of the total number of people tested, and $53.1 \%$ of the total number of reactive tests. MSM accounted for $57.2 \%$ of all people tested, and $73.58 \%$ of the total number of reactive tests.

Figure 3 describes the contribution of each transmission group to the total number of people tested, and the total number of reactive tests between 1995 and 2018. It shows that MSM as a proportion of all people tested has increased greatly over time, reaching $74.7 \%$ $(7,988 / 10,700)$ in 2018 . The opposite trend is visible among PWID. Each year between 1996 and 2004, PWID were the transmission group with the highest number of reactive tests. Since 2005 , the proportion of this group has gradually diminished, reaching the lowest value $(0.7 \% ; 71 / 10,700)$ in 2018 . In the same period, the proportion of all reactive tests for MSM (MSM plus 


\section{FIGURE 4}

Percentage of HIV reactive tests by transmission group per year, Catalonia, Spain, 1995-2018 (n people tested = $106,992^{\mathrm{a}} ; \mathrm{n}$ reactive tests $=2,848^{\mathrm{b}}$ )

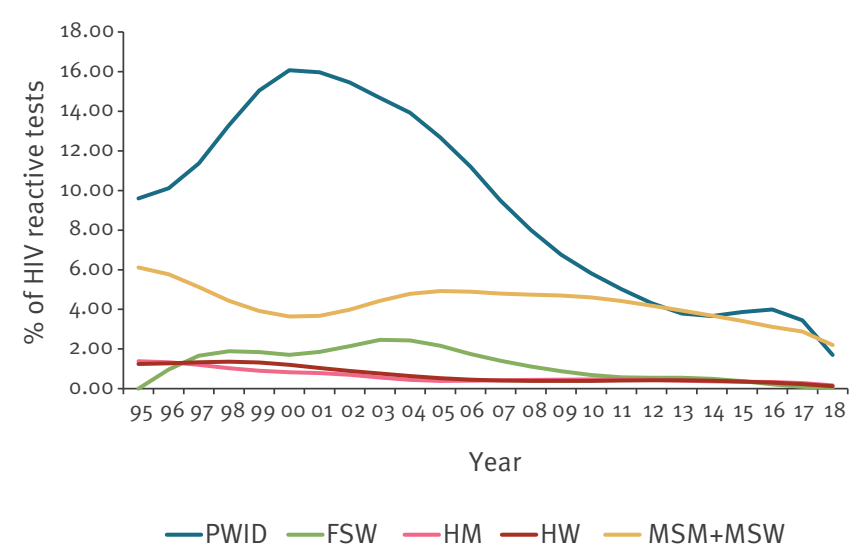

DEVO: voluntary detection; FSW: female sex worker; HM: heterosexual men; HW: heterosexual women; MSM: men who have sex with men; MSW: male sex worker; PWID: people who inject drugs.

a Only people tested within the DEVO network were included. Before 2014 disaggregated data refers to number of tests instead of people tested.

${ }^{b}$ Only HIV reactive tests performed within the DEVO network were included.

The lines were smoothed using the moving average method.

MSW) increased, accounting for $95.0 \%$ of the total number of reactive tests detected in 2018.

The highest reactivity rate in each transmission group during the whole period of study was found in PWID (ranging between $1.1 \%(1 / 95)$ in 2014 and $25.0 \%$ (8/32) in 2000), followed by MSM plus MSW (ranging between $2.2 \%(181 / 8,209)$ in 2018 and $10.2 \%(17 / 167)$ in 1995) (Figure 4). Nevertheless, in recent years the reactivity rate in the PWID group has gradually decreased. This decrease is not statistically significant, due to the low number of PWID tested. The reactivity rate in MSMplusMSW has shown a statistically significant decrease $(p<0.001)$, especially in the past 10 years, reaching $2.2 \%(181 / 8,209)$ in 2018 . For the rest of the groups, no significant trend was observed.

Figure 5 shows the increase of the contribution of HIV POCT in the community to the overall total number of cases registered in the Catalonia HIV registry. The percentage of positive cases in the Catalonia HIV registry which were first detected in the DEVO network has gradually increased, from $4.5 \%$ (34/763) in 2001 to $37.9 \%(219 / 578)$ in 2017 . In the case of MSM plus MSW, this contribution is higher, reaching $70.0 \%$ of total HIV diagnosed cases in the Catalonia HIV registry among MSM in 2018.

In 2018 , a total of 14,537 tests were performed in the DEVO network on 10,894 individuals, of which $1.8 \%$
(191/10,894) were reactive. Of these reactive tests, $94.8 \%(181 / 191)$ had a confirmatory test, of which $100 \%$ were confirmed as positive. Of the total number confirmed positive, 93.9\% (170/181) were linked to care. The percentage of individuals with a reactive screening test who were linked to care was $89.0 \%$ (170/191). This percentage has not varied considerably since these data were available $(81.7 \%$ in 2014 , $95.0 \%$ in $2015,89.5 \%$ in 2016 , and $92.7 \%$ in 2017 ).

\section{Discussion}

This study shows the contribution of community-based HIV POCT in improving early HIV diagnosis in Catalonia over time, especially among key populations, and demonstrates that the collected data are an important source of strategic information to be included into the Integrated AIDS/HIV/STI Surveillance System of Catalonia (SIVES).

In Catalonia, community-based HIV testing has been monitored and has formed part of HIV Surveillance since 1995. The DEVO Network has made it possible to collect standardised data on each person tested in CBVCT services. The collected data complement strategic information on key populations and thus make it possible to improve HIV prevention strategies aimed at these key populations. The continual monitoring performed by the DEVO Network has improved public health decision-making at the Public Health Agency of Catalonia by detecting changes in HIV testing uptake, in HIV tester profiles and in HIV test-seeking behaviours [5].

The DEVO Network succeeded in scaling up HIV testing among key populations, with the number of tests performed in the year following the implementation of POCT increasing by $103 \%$ from the previous year [5].

HIV POCT have the potential to increase the number of people who know their HIV status [24]. The POCT that meet the WHO's ASSURED (affordability, sensitivity, specificity, user-friendly, rapid and robust, equipmentfree and deliverable) criteria [25] follow a simple procedure involving a limited number of steps and are equipment-free, ensuring they can be performed outside traditional laboratory settings by staff with no formal laboratory training [24]. Additionally, both providers and clients prefer rapid tests over traditional tests $[26,27]$. Several studies have shown the efficacy of CBVCT strategies using HIV POCT to improve HIV testing uptake in populations at higher risk of exposure to HIV $[6,7,9]$. The DEVO Network has shown to be successful in providing testing to at-risk populations. In the period $1995-2018,57.2 \%$ of tests were performed on MSM, $7.6 \%$ on SW (FSW plus MSW), $2.6 \%$ on PWID, $1.5 \%$ on transgender people and $45.2 \%$ on foreign nationals (including migrant population). A recent study showed that in Catalonia, $12.3 \%$ of those living with HIV were still undiagnosed, and this proportion was higher in migrants [28]. Therefore, in Catalonia, providing access to HIV POCT in the community is 


\title{
FIGURE 5
}

Number of HIV reactive screening tests from the DEVO network, total number of cases registered in the Catalonia HIV registry and the percentage ${ }^{\mathrm{a}}$ of tests recorded in the Catalonia HIV registry first detected in the DEVO network for (A) all people tested ( $\mathrm{n}=2,483$ in DEVO network, $\mathrm{n}=13,004$ in HIV registry) and (B) men who have sex with men and male sex workers only ( $\mathrm{n}=2,099$ in DEVO network, $\mathrm{n}=5,660$ in HIV registry), Catalonia, Spain, 2001-2017

\section{A. All people tested}

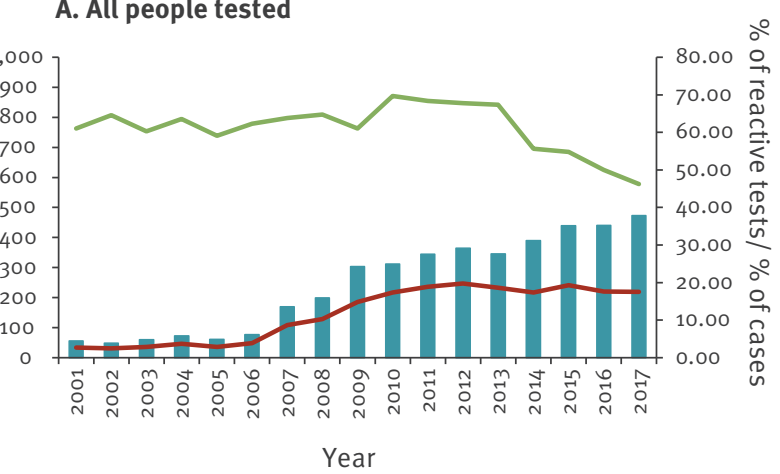

Percentage of Catalonia HIV

registry cases first detected in DEVO
- HIV reactive tests from DEVO
B. MSM + MSW

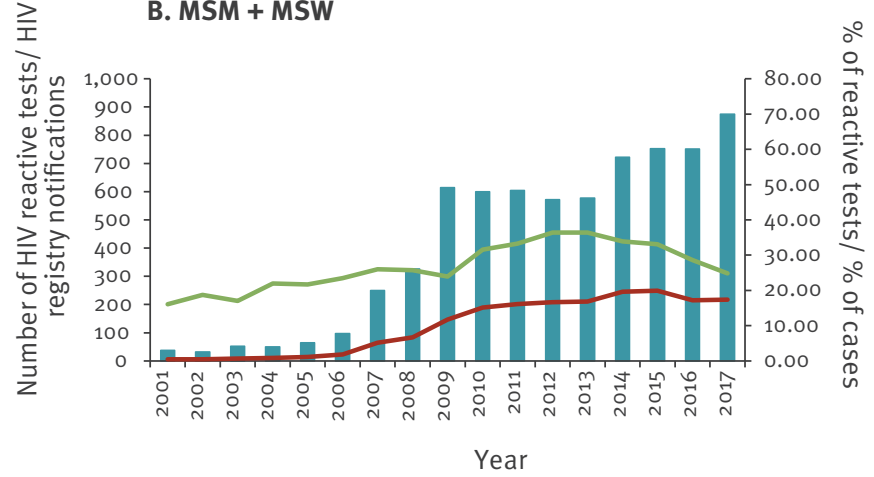

— All Catalonia HIV registry cases

DEVO: voluntary detection; MSM: men who have sex with men; MSW: male sex worker.

\begin{abstract}
a Percentage calculated as number of reactive tests obtained by DEVO community testing divided by number of total cases recorded in the Catalonia HIV registry.
\end{abstract}

important, especially for populations facing barriers to accessing the healthcare system, such as the migrant population.

The low number of PWID tested in the DEVO network can be explained by the fact that most PWID are tested in harm reduction centres. In Catalonia there is a network of harm reduction programmes run by mobile units, street teams or facility-based centres. The facility-based centres are located in areas of drug trafficking and drug consumption, or in drug treatment clinics [29].

Linkage to care and treatment for those with a reactive test in the DEVO network is high (89\%). A recent systematic review and meta-analysis of studies in the WHO European Region [30] showed a pooled estimate of $85 \%$ (95\% Cl: $75-93)$ of people with reactive tests linked to care within 3 months. Linkage of those with a reactive test to appropriate specialist services is a key step in the HIV continuum of care, as immediate initiation of treatment has substantial benefits in reducing the risk of patient morbidity, as well as reducing onward transmission [30].

In the past 10 years, a statistically significant decreasing trend has been observed in the percentage of MSM with a HIV reactive test. This trend could be explained by the success of different strategies of combined prevention in this key population, including increased testing frequency and earlier initiation of HIV treatment. BCN Checkpoint (the CBVCT service with the largest HIV testing activity, particularly among MSM) has gone further to promote earlier initiation of HIV treatment by introducing qualitative PCR POCT for the detection of acute HIV infection [31]. This, coupled with their preexposure prophylaxis (PrEP) service in the framework of research studies, has broadened the portfolio of preventive services available to users of the Checkpoint. In England, the incidence of new HIV infections in MSM attending sexual health clinics fell by 55\% in 2016 and 2017 [32,33], and was attributed to an increase in HIV testing, earlier initiation of HIV treatment and the scale up of privately purchased generic PrEP in England from late 2017 onwards.

In Catalonia, universal treatment (treatment independent of $\mathrm{CD}_{4}{ }^{+}$cell count for patients newly diagnosed with HIV) has had a positive impact on the dynamics of the viral load in people living with HIV [34]. This, along with increasing testing and linkage to care as part of a combined prevention strategy, can explain the decrease in the percentage of new HIV diagnoses in the DEVO network. The increase in number of sites offering HIV testing thanks to the introduction of HIV POCT has increased the proportion of community detected HIV cases in the overall number of HIV cases reported in Catalonia, increasing from $4.5 \%$ in 2001 to $37.9 \%$ in 2017. The impact of introducing HIV POCT was even larger for MSM, where $70 \%$ of all new HIV diagnoses in 2017 were diagnosed in the community setting. This suggests that CBVCT services are a valuable element of the strategy to increase HIV testing in Catalonia, especially for MSM. These estimations are higher than that presented in a 2019 study showing that in several southern European countries, $0.2-19.7 \%$ of total HIV cases and $0.5-37.0 \%$ of HIV cases among MSM were diagnosed through CBVCT services [35]. 
The longstanding experience of the DEVO Network and its results has been used as a basis for establishing the COBATEST Network, a European network of CBVCT services that share standardised data $[8,14]$.

There are a number of limitations to this study. Firstly, the disaggregated data presented in the Table from 1995 to 2013 refers to the number of tests performed, while data from 2014 to 2018 refer to the total number of people tested. This is due to improvements made to the data collection system and its integration into the COBATEST network. After 2013 a unique identifier was assigned to each client, allowing detection of repeat testers, and at the same time ensuring the anonymity of people tested. This could have led to an under-estimation of the number of tests in the period 2014-2018, especially in the MSM group as MSM are more often repeat testers. Secondly, the number of HIV positive cases from1995 to 2007 refers to HIV confirmed cases only, as the test offered was the conventional laboratory test. Since the introduction of HIV POCT, the number of HIV positive cases refers to reactive cases as in some cases, the information related to referral and confirmation of the diagnosis is not complete. So the number of HIV reactive cases in the period 1995-2006 could be higher. Lastly, regarding the number of cases detected in the DEVO network as a percentage of the total number of Catalonia HIV registry cases - as it was assumed that all reactive cases were linked to care and therefore were added to the Catalonia HIV registry, the contribution could have been overestimated.

\section{Conclusion}

In conclusion, our study with a monitoring series of almost 25 years reinforces the important role that community-based HIV POCT has on the improvement of early HIV diagnoses in key populations, and highlights the importance of monitoring these data and including them in a regional or national HIV surveillance system. To ensure sustainability of the community testing services, key stakeholders must commit to including CBVCT services in the design and plan for strategies to achieve the 90-90-90 objectives.

\section{Acknowledgements}

We are grateful to all the DEVO network services and their teams for their continuous work collecting and providing HIV testing data: Jorge Saz, Michael Meulbroek, Ferran Pujol, Félix Pérez, Albert Dalmau-Bueno (BCN Checkpoint); Comisión de Prueba Rápida de VIH y otras ITS (Stop Sida); Jaime Quezadas, Gemma Ricomà (ACASC); Jordi Baroja, Mari Paz Oliver, Georgina Picas, Erika Ros, Maria Ferrero Barrio, Jacque Segura I Mireia Aldavert (CJAS); Anna Rafel (Associació Antisida Lleida); Ander Pazos, William Mejías, Joaquim Roqueta (Gais Positius); Anna Avellaneda, Elena Griñán, Júlia Collignon, Alberto Capitán (Actuavallès); Mercè Meroño, Cati Fons, Marisa Cebrián (Àmbit Prevenció); Lorena Andreo, Víctor Navarro, Himalaya Vallejo, Sara Riba (CAS/ ARD Lluís Companys, CreuRoja Barcelona); Lluís Romero (ASSEXORA'T); Anna Lara, Lluís Franch (ACAS Girona); Araceli Otón, (Creu Roja Tarragona).
Conflict of interest

None declared.

\section{Authors' contributions}

LFL led the data analysis and drafting of the manuscript supported by JRU, AC and JCa. JS, AM, JQ, JB, AR, AP, AA, MM, $\mathrm{LA}, \mathrm{LR}, \mathrm{AL}, \mathrm{AO}$ provided HIV testing data. BR, RM and JCo contributed to the development of the different drafts. All authors commented on various drafts of the manuscript and approved the final version.

\section{References}

1. Tavoschi L, Hales D. Monitoring of HIV testing services in the EU/EEA. Euro Surveill. 2016;21(48):30410. https://doi. org/10.2807/1560-7917.ES.2016.21.48.30410 PMID: 27934584

2. World Health Organization (WHO). Consolidated guidelines on person-centred HIV patient monitoring and case surveillance. Geneva: WHO; 2017. Available from: https://www.who.int/hiv/ pub/guidelines/person-centred-hiv-monitoring-guidelines/en/

3. Centre d'Estudis Epidemiològics sobres les Infeccions de Transmissió Sexual i Sida de Catalunya (CEEISCAT). Vigilànciaepidemiològica de la infecciópel VIH i la SIDA a Catalunya. Informeanual 2017. [Epidemiological surveillance of HIV and AIDS infection in Catalonia. Annual report 2017]. Badalona: CEEISCAT; 2018. Catalan. Available from: https:// canalsalut.gencat.cat/web/.content/_A-Z/S/sida/enllasos/ anual_VIH.pdf

4. Centre d'Estudis Epidemiològics sobres les Infeccions de Transmissió Sexual i Sida de Catalunya (CEEISCAT). Epidemiological Report SIVES 2015.Technical document $\mathrm{n}-22$, Integrated Surveillance System for STI and HIV in Catalonia. Badalona; CEEISCAT; 2015. Available from: https://salutpublica.gencat.cat/web/.content/minisite/ aspcat/vigilancia salut publica/vih-sida-its/05_CEEISCAT/ publicacions-cientifiques/informes/SIVES/SIVES_2015/ sives2015_ENG.pdf

5. Fernàndez-Lopez L, Rifà $B$, Pujol F, Becerra J, Pérez $M$, Meroño $M$, et al. Impact of the introduction of rapid HIV testing in the Voluntary Counselling and Testing sites network of Catalonia, Spain. Int J STD AIDS.2010;21(6):388-91. PMID: 20606218

6. Suthar AB, Ford N, Bachanas PJ, Wong VJ, Rajan JS, Saltzman AK, et al. Towards universal voluntary HIV testing and counselling: a systematic review and metaanalysis of community-based approaches. PLoS Med. 2013;10(8):e1001496. https://doi.org/10.1371/journal. pmed.1001496 PMID: 23966838

7. Pottie K, Medu O, Welch V, Dahal GP, Tyndall M, Rader T, et al. Effect of rapid HIV testing on HIV incidence and services in populations at high risk for HIV exposure: an equity-focused systematic review. BMJ Open. 2014;4(12):eoo6859. https:// doi.org/10.1136/bmjopen-2014-006859 PMID: 25510889

8. Fernàndez-LópezL. Reyes-UrueñaJ, AgustíC, KustecT, KlavsI, CasabonaC; COBATEST Network group. The COBATEST network: a platform to perform monitoring and evaluation of HIV community-based testing practices in Europe and conduct operational research. AIDS Care. 2016;28(February) Suppl 1;32-6. . Available from: http://www.tandfonline.com/doi/full/1 $0.1080 / 09540121.2016 .1146218$

9. Fernàndez-LópezL. Reyes-UrueñaJ, AgustíC, KustecT, SerdtM, Klavsl, et al.The COBATEST network: monitoring and evaluation of HIV community-based practices in Europe, 2014-2016. HIV Med. 2018;19(Suppl 1):21-6. PMID: 29488701

10. European Centre for Disease Prevention and Control (ECDC). Thematic report: continuum of HIV care. Monitoring implementation of the Dublin Declaration on partnership to fight HIV/AIDS in Europe and Central Asia: 2017 progress report. Stockholm: ECDC; 2017: Available from: https://www. ecdc.europa.eu/sites/default/files/documents/Continuum-ofHIV-care-2017.pdf

11. Sulat JS, Prabandari YS, Sanusi R, Hapsari ED, Santoso B. The impacts of community-based HIV testing and counselling on testing uptake. J Heal Res.2018;32(2):152-63. https://doi. org/10.1108/JHR-01-2018-015

12. Cohen MS, Chen YQ. McCauleyM, GambleT, HosseinipourMC, KumarasamyN, et al. Prevention of HIV-1 infection with early antiretroviral therapy. N Engl J Med. 2011;365(6):493-505. PMID: 21767103

13. Sullivan AK, Sperle I, Raben D, Amato-Gauci AJ, Lundgren JD, Yazdanpanah Y, et al. HIV testing in Europe: Evaluating the 
impact, added value, relevance and usability of the European Centre for Disease Prevention and Control (ECDC)'s 2010 HIV testing guidance. Euro Surveill. 2017;22(48):17-00323. PMID: 29208158

14. Fernàndez-López L, Reyes-Urueña J, Agustí C, Kustec T, Serdt $M$, Klavs I, et al. The COBATEST network: monitoring and evaluation of HIV community-based practices in Europe, 20142016.HIV Med. 2018;19(Suppl 1):21-6. PMID: 29488701

15. HIV-COBATEST Project. Core indicators to monitor community based voluntary counselling and testing (CBVCT) for HIV Guidelines for CBVCT services. Field-test version. 2012. Available from: https://eurohivedat.eu/arxius/ehe cdocsmenu_cdocsmenu_doc_3-CBVCT_core_indicators_field_ test_version.pdf

16. Joint United Nations Programme on HIV/AIDS (UNAIDS). Global AIDS Response progress reporting: monitoring the 2011 political declaration on HIV/AIDS: guidelines on construction of core indicators: 2012 reporting. Geneva: UNAIDS; 2011. Available from: https://unaids-test.unaids.org/sites/default/ files/unaids/contentassets/documents/document/2011/ JC2215_Global_AIDS_Response_Progress_Reporting_en.pdf

17. World Health Organization (WHO). Guide for monitoring and evaluating national HIV testing and counselling (HTC) programmes: field-test version. Geneva: WHO; 2011. Available from: https://apps.who.int/iris/bitstream/ handle/10665/44558/9789241501347 eng.pdf;jsessionid =2AD C244Fo85B39203050FA5C20C1A9FE?sequence =1

18. European Centre for Disease Prevention and Control (ECDC). Implementing the Dublin Declaration on partnership to fight HIV/AIDS in Europe and Central Asia: 2010 progress Report. Stockholm: ECDC; 2010. Available from: https://www.ecdc. europa.eu/sites/default/files/media/en/publications/ Publications/100720_implementing_dublic_declaration_hiv_ aids_2010_progress_report.pdf

19. Abbott. ALERE Determine HIV-1/2 package insert. 2011. [Accessed: 23 Jan 2020]. Available from: https://www. globalpointofcare.abbott/en/product-details/determinehiv-1-2.html

20. Mayhood MK, Afwamba IA, Odhiambo CO, Ndanu E, Thielman NM, Morrissey AB, et al. Validation, performance under field conditions, and cost-effectiveness of Capillus HIV-1/ HIV-2 and determine HIV-1/2 rapid human immunodeficiency virus antibody assays using sequential and parallel testing algorithms in Tanzania. J Clin Microbiol. 2008;46(12):3946-51. https://doi.org/10.1128/JCM.01045-08 PMID: 18923015

21. Abbott. Alere Determine HIV-1/2 Ag/Ab Combo Serum / Plasma Procedure Product Demo (English). Scarborough: Abbott; 2019. [Accessed: 23 Jan 2020]. Available from: https://www. globalpointofcare.abbott/en/product-details/determine-1-2-agab-combo.html.

22. Carlberg C. More predictive analytics: Microsoft Excel. Indianapolis: QUE; 2016.

23. Dahl A. Optimal linkage to care among MSM: a practical guide for CBVCT's and points of care. Barcelona: Euro HIV EDAT; 2017. Available from: https://eurohivedat.eu/arxius/ehe docsmenu docsmenu_doc_128-20131101_D05_00_OTH_1_EN_PS.pdf

24. Branson BM. Point-of-Care Rapid Tests for HIV Antibodies. PatientennaheSchnelltests fur den Nachweis von HIVAntikorpern. Lab Med. 2003;27(7-8):288-95. Available from: http://doi.wiley.com/10.1046/j.1439-0477.2003.03049.x

25. Peeling RW, Holmes KK, Mabey D, Ronald A. Rapid tests for sexually transmitted infections (STIs): the way forward. Sex Transm Infect. 2006;82(Suppl 5):v1-6. https://doi.org/10.1136/ sti.2006.024265 PMID: 17151023

26. Conway DP, Guy R, Davies SC, Couldwell DL, McNulty A, Smith $\mathrm{DE}$, et al. Rapid HIV testing is highly acceptable and preferred among high-risk gay and bisexual men after implementation in Sydney sexual health clinics. New South Wales. 2015.

27. Conway DP, Guy R, McNulty A, Couldwell DL, Davies SC, Smith $D E$, et al. Effect of testing experience and profession on provider acceptability of rapid HIV testing after implementation in public sexual health clinics in Sydney. HIV Med. 2015;16(5):280-7. PMID: 25604470

28. Reyes-Urueña JM, Campbell CNJ, Vives N, Esteve A, Ambrosioni J, Tural C, et al. Estimating the HIV undiagnosed population in Catalonia, Spain: descriptive and comparative data analysis to identify differences in MSM stratified by migrant and Spanishborn population. BMJ Open. 2018;8(2):e018533. PMID: 29490955

29. Fernàndez-López L, Folch C, Majó X, Gasulla L, Casabona J, Fernandez-LopezL. Implementation of rapid HIV and HCV testing within harm reduction programmes for people who inject drugs: a pilot study. AIDS Care. 2016;28(6):712-6. https://doi.org/10.1080/09540121.2016.1164290 PMID: 27007000
30. Croxford S, Yin Z, Burns F, Copas A, Town K, Desai S, et al. Linkage to HIV care following diagnosis in the WHO European Region: A systematic review and meta-analysis, 2006-2017. PLoS One. 2018;13(2):e0192403. . Available from: http://www. ncbi.nlm.nih.gov/pubmed/29451875 https://doi.org/10.1371/ journal.pone.0192403 PMID: 29451875

31. Meulbroek M, Dalmau-Bueno A, Pujol F. Adjusted criteria for point-of-care HIV-RNA testing leads to improved detection of acute HIV infection. HIV Research for Prevention (HIVR4P) 2018; 21-25 October 2018, Madrid, Spain. Abstract Po603.

32. Ogaz D, Miltz AR, Desai S, Saunders J, Charlett A, Gill ON, et al. Preparing for PrEP in England: Prevalence and incidence of HIV and bacterial STIs. Conference on Retroviruses and Opportunistic Infections (CROI); 4-7 March 2019, Seattle, United States. Abstract 48. Available from: http://www. croiconference.org/sessions/preparing-prep-englandprevalence-and-incidence-hiv-and-bacterial-stis

33. Nwokolo N, Hill A, McOwan A, Pozniak A. Rapidly declining HIV infection in MSM in central London. Lance HIV. 2017;4(11):e482-3. https://doi.org/10.1016/S23523018(17)30181-9 PMID: 29066095

34. Reyes-Urueña J, Montoliu A, Bruguera A, Miró JM, Podzamczer $D$, Domingo $P$, et al. Impact of universal treatment on the dynamics of viral load, Catalonia and the Balearic Islands. PISCIS cohort. 23rd International Workshop on HIV and Hepatitis Observational Databases (IWHOD). 28-30 March 2019, Athens, Greece. Available from: https://www. researchgate.net/project/PISCIS-Cohort/update/5C9ce918384 3bo342433f9af.

35. Rocha M, Pujol Roca F, SazBerges J, Chanos S, Polkas G, Mattioli $S$, et al. High contribution, low public funding: Southern European Checkpoints' role in HIV detection in 4 countries. HepHIV2019 Bucharest Conference: Challenges of Timely and Integrated Testing and Care; $28-30$ January 2019, Bucharest, Romania. Available from: https://www. eurotest.org/Portals/o/PS1_1_High\%2ocontribution $\% 2 \mathrm{C} \% 20$ low $\% 2$ opublic $\% 2$ ofunding $\% 20$ Southern $\% 2$ European $\% 20$ Checkpoints\%27\%20role\%20in\%20HIV\%2odetection $\% 20$ in $\% 204 \% 20$ countries.pdf

\section{License, supplementary material and copyright}

This is an open-access article distributed under the terms of the Creative Commons Attribution (CC BY 4.0) Licence. You may share and adapt the material, but must give appropriate credit to the source, provide a link to the licence and indicate if changes were made.

Any supplementary material referenced in the article can be found in the online version.

This article is copyright of the authors or their affiliated institutions, 2020. 